\title{
T2K on-axis detector results: cross section measurements
}

\author{
Benjamin Quilain ${ }^{1, a}$ on behalf of the T2K collaboration \\ ${ }^{1}$ Ecole Polytechnique, IN2P3-CNRS, Laboratoire Leprince-Ringuet, Palaiseau, France
}

\begin{abstract}
The T2K experiment brings the world leading constraints on the atmospheric parameters, $\sin ^{2} \theta_{23}=0.514 \pm 0.082$ and $\left|\Delta m_{32}^{2}\right|=\left(2.44_{-0.15}^{+0.17}\right) \times 10^{-3} \mathrm{eV}^{2} \cdot \mathrm{c}^{-4}$, along with first observation of the electron neutrino appearance in the muon neutrino beam. In the upcoming years, a higher precision measurement of the PMNS matrix parameters with possible evidence of a non zero $\mathrm{CP}$ violation phase requires a reduction in the main systematics which come from the cross section model. The INGRID detector is the onaxis detector of the long-baseline T2K experiment. Though its main goal is to monitor the neutrino flux intensity and off-axis angle, this proceeding presents the $v_{\mu}$ cross section measurements that have been performed to reduce the uncertainty on the neutrino cross section models.
\end{abstract}

\section{Introduction}

$\mathrm{T} 2 \mathrm{~K}$ is a long-baseline neutrino experiment with the main goal to provide the reactor mixing angle $\theta_{13}$ by observing $v_{\mu} \rightarrow v_{\mathrm{e}}$ oscillations. A non zero $\theta_{13}$ has been observed at T2K [1]. When combined with precise reactor measurements [2] [3], the $v_{\mu} \rightarrow v_{\mathrm{e}}$ observations also constrains the leptonic CP violation phase value, $\delta_{C P}$. The very high oscillated $v_{e}$ rate observed in T2K particularly leads to a hint of a maximal CP violation phase, $\delta_{C P}=270^{\circ}$ [1]. On top of this, T2K is designed to provide accurate measurement of the atmospheric parameters, $\theta_{23}$ and $\Delta m_{32}^{2}$, through the measurement of muon neutrino disappearance [4].

In the upcoming years, the refinement of these measurements requires a decrease in the current systematic error on these two results. In both the appearance and disappearance channels, the dominant systematic errors come from the cross section model uncertainties, that are necessary to extrapolate the neutrinos fluxes at Super-Kamiokande from ND280 measurements. In this proceeding, the cross section measurements provided by the on-axis INGRID detector are presented. The measurement of the $v_{\mu}$ charged current inclusive cross section on iron and carbon is first shown. Then, two exclusive measurements are tackled. The cross section of $v_{\mu}$ interaction through quasi-elastic charged current on carbon is presented. Then, the search for coherent pion production is shown. Finally, the upcoming improvements of these measurements are briefly mentioned.

\section{The T2K experiment and the on-axis detector}

A high purity $v_{\mu}$ beam is produced using the $30 \mathrm{GeV}$ proton accelerator complex at J-PARC and detected $295 \mathrm{~km}$ away in the Super-Kamiokande far detector. The neutrino flux is directed $2.5^{\circ}$ from

\footnotetext{
a e-mail: benjamin.quilain@1lr.in2p3.fr
} 

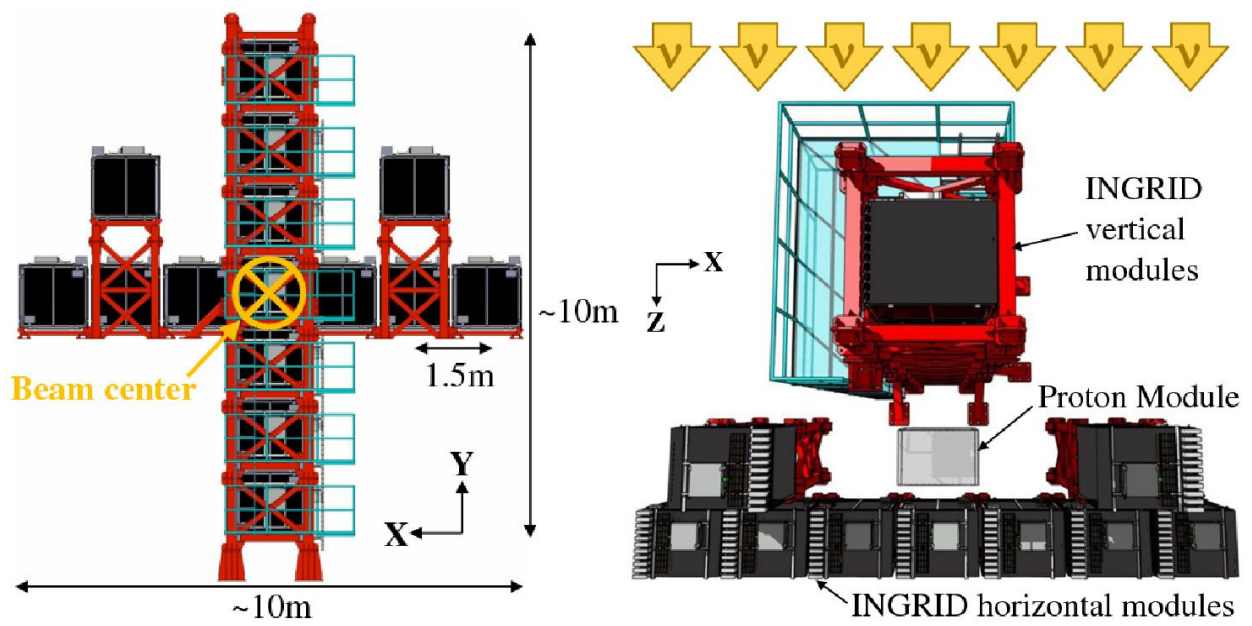

Figure 1. The INGRID front (left) and upper (right) view from the on-going neutrino beam.

Super-Kamiokande, in order to provide a narrow band $v_{\mu}$ spectrum peaked at $600 \mathrm{MeV}$. This offaxis technique maximises the number of oscillated neutrinos and reduces the background from non oscillating $v_{\mu}$ and beam intrinsic $v_{e}$. The oscillation measurement requires an accurate knowledge of these neutrino fluxes. Hence, these fluxes are monitored prior to standard oscillations by a near detector complex located $280 \mathrm{~m}$ away from the T2K target. The ND280 detector is located $2.5^{\circ}$ off-axis and constrains the different neutrino fluxes. The INGRID detector is located on-axis, and monitors the $v_{\mu}$ flux intensity and measures the beam central position to accurately determine the offaxis angle with a precision below $1 \mathrm{mrad}$. In order to do so, the INGRID detector is mainly composed of fourteen modules assembled in a cross shape structure, as shown on Figure 1. On top of this, two shoulder modules are added to monitor the beam shape asymmetry. Each of these sixteen modules is composed of a sandwich of 11 scintillator tracking planes and iron planes (nine $6.5 \mathrm{~cm}$ thickness) that constitutes most of the interacting material. Each tracking plane is composed of a vertical and horizontal scintillator plane, each composed of 24 scintillators, having dimensions of $120 \times 5 \times 1 \mathrm{~cm}^{3}$. A seventeenth module called the Proton Module (PM) has been installed between the horizontal and vertical modules, in order to specifically measure cross section. The PM is composed of 18 vertical and 18 scintillator planes, that are alternately disposed. They are separated by $2.3 \mathrm{~cm}$ of air. Each of the tracking plane is composed of 32 scintillators of two different dimensions. On each edge of the scintillator planes are eight INGRID scintillators. The sixteen most central scintillators of each plane have a smaller width (dimensions of $120 \times 2.5 \times 1.3 \mathrm{~cm}^{3}$ ) to increase the detector granularity.

\section{Measurement of the $v_{\mu}$ cross section through inclusive charged-current interaction.}

The signal for neutrino oscillation is only constituted of CC interactions occuring in ND280 and Super-Kamiokande. In this context, the measurement of the inclusive CC cross section brings an important contribution to the reduction of systematics on oscillation analyses. The INGRID detector is designed to detect the outgoing leptons from neutrinos interacting through charged current interactions 


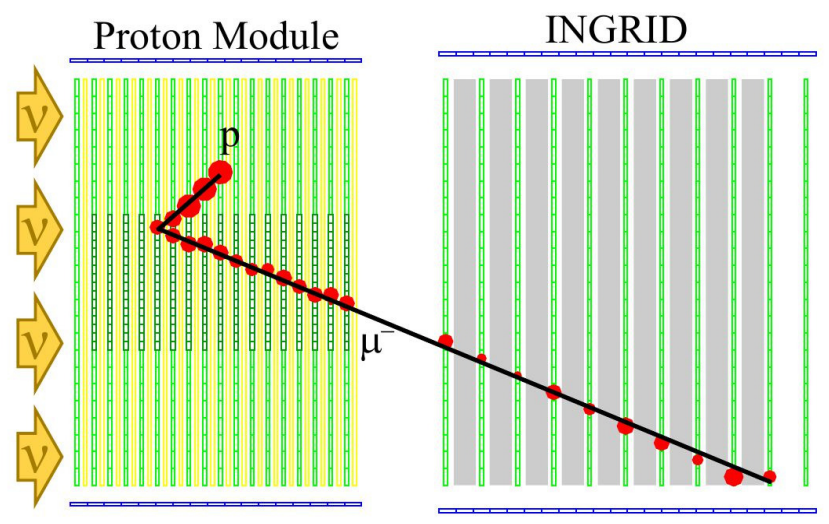

Figure 2. An event display of a charged current quasi-elastic event occuring in the PM. One observes the muon propagates into the downstream INGRID module.

(CC), and therefore is a good candidate to measure this cross section. Therefore, the measurement of the flux averaged cross section will be perfomed on two different targets. The CC cross section on INGRID iron (INGRID central horizontal module only) and on PM hydrocarbon (scintillator) targets will be measured independently, before showing the ratio of the measurements. It will be observed than the latter further constrains the cross section models due to reduction of the main systematics coming from the flux uncertainties. A simulated charged current interaction occuring in the PM is shown on Figure 2.

The CC cross section is defined as:

$$
\sigma_{C C}=\frac{N_{\text {sel }}-N_{\mathrm{BG}}}{\phi N_{T} \epsilon}
$$

where $N_{\text {sel }}$ and $N_{\mathrm{BG}}$ respectively stand for the number of selected and background events. $\phi$ is the total neutrino flux intercepting the interacting material, $N_{T}$ is the number of target nucleons and $\epsilon$ is the selection efficiency.

\subsection{Event selection}

The CC interaction selection is based on the identification of the associated lepton track, namely the $\mu$. A first hit cluster selection is applied to remove electronic noise. It is based on the combination of four hits into a $100 \mathrm{~ns}$ time window and at least three active tracking planes (w/ one vertical + horizontal hit). Then, the two dimensional track reconstruction is applied for each module independently, before being matched on geometrical criteria between the different modules. For the PM, it is required that at least one track continues into the downstream INGRID module, as for the $\mu$ on Figure 2. For a standard INGRID module, there is no downstream module to match tracks. Therefore, an additional acceptance cut that mimics a downstream module is applied. Two dimensional tracks are then assembled into three dimensional tracks which are themselves gathered depending on their upstream vertex. Finally, the cosmic muon background is removed applying a $\pm 100 \mathrm{~ns}$ time cut around the expected beam arrival, a veto and a fiducial volume cuts. This reconstruction and selection are applied in the PM and INGRID, and provide a CC purity of $89.4 \%$ and $88.8 \%$ respectively, with respective $41.2 \%$ and $42.9 \%$ efficiencies. 
This selection is applied on data, which corresponds to the $6.04 \times 10^{20}$ protons on target (POT) taken when the PM and INGRID were both installed. The MC is used to evaluate the selection efficiency $\epsilon$, and to determine the background $N_{B G}$, mainly composed of NC interactions and sand muons. The neutrino flux $\phi$ is determined for each neutrino type using the T2K flux simulation. A dominant $v_{\mu}$ contribution $(>95 \%)$ is observed, with a relatively small contamination from $\bar{v}_{\mu}(4 \%)$ and $<1 \%$ contamination from $v_{e}$. Finally, the number of target nucleons $N_{T}$ is evaluated both knowing the elemental composition of each module (Standard INGRID module or PM) and weighting it. The summary of each quantity required to evaluate the cross section is shown in Table 1. One observes the number of events in the INGRID standard module is 14 times higher than for the PM, and so is the number of target nucleons.

\begin{tabular}{|c|c|c|c|c|c|}
\hline \hline & $N_{\text {sel }}$ & $N_{\text {BG }}$ & $\phi$ & $N_{T}$ & $\epsilon$ \\
\hline$\sigma_{C C}^{\mathrm{Fe}}$ & 523045 & 67838 & $2.999 \times 10^{13} \mathrm{~cm}^{-2}$ & $2.461 \times 10^{30}$ & $42.6 \%$ \\
$\sigma_{C C}^{\mathrm{CH}}$ & 36330 & 5385.5 & $3.025 \times 10^{13} \mathrm{~cm}^{-2}$ & $1.799 \times 10^{29}$ & $41.2 \%$ \\
\hline \hline
\end{tabular}

Table 1. Summary of the candidate events that interacted through charged boson exchange in iron (INGRID) and hydrocarbon (PM). The selected background $N_{\mathrm{BG}}$, the intercepting neutrino flux in the detector fiducial volume $(\phi)$, the number of nucleon targets (T) and the efficiency $\epsilon$ are also indicated for the two detectors.

\subsection{Systematic errors}

The systematic error is evaluated coming from three independent sources: the neutrino flux, the interaction model and the detector response. For each source, the impacts on $N_{B G}, \phi, N_{T}$ and $\epsilon$ are evaluated.

- Neutrino flux uncertainties: The neutrino flux uncertainties can be divided into two categories: the error on hadron production and on the $\mathrm{T} 2 \mathrm{~K}$ beamline. The hadron production is driven by the NA61/SHINE measurements [5][6] and the Eichten and Allaby data [7][8]. The T2K beamline uncertainty is related to operatial variations in the beamline condition such as the off-axis angle, the horn current. The method to estimate these uncertainties is described in [9].

- Neutrino interaction uncertainties: The interaction on INGRID and PM targets are simulated using the T2K NEUT generator. The associated uncertainties are evaluated using a data-driven method. NEUT predictions are compared to existing external neutrino-nucleus data in the T2K energy range. Some parameters are fitted such as the axial mass and some ad-hoc paramaters are introduced often with large uncertainties to take into account remaining discrepancies between NEUT and the external data like [10],[11]. The method used to evaluate these uncertainties is described in [4].

- INGRID and PM detector response: The uncertainties from the detector come from different sources: target mass measurement, variations of the MPPC dark rate during data taking, discrepancy between detection efficiency between the MC and data. A lot of these systematic errors are evaluated using an independent sample constituted by the "sand muons" coming from neutrino interactions in the surrounding ND280 pit.

The systematic errors are summarised in Table 2. One observes the absolute measurement on hydrocarbon and ratio are associated with a $\sim 10 \%$ error, which predominantly comes from flux errors. The neutrino interaction errors aren't negligible, though siginificantly smaller (4\%) and the same goes for the detector errors $(<2 \%)$. The interest of the relative measurement on the iron to 
carbon ratio is most of the dominant systematics are washed out. One observes most of the flux errors become subdominant for this measurement, and the neutrino interaction are highly reduced (to $1.6 \%$ ). Therefore, the detector response errors dominate for the relative measurement, and the total error is reduced to $<3 \%$.

\begin{tabular}{|c|c|c|c|}
\hline Source & $\sigma_{C C}^{F e}$ & $\sigma_{C C}^{C H}$ & $\overline{\sigma_{C C}^{F e} / \sigma_{C C}^{C H}}$ \\
\hline Neutrino flux & $\begin{array}{l}12.7 \% \\
-10.3 \%\end{array}$ & $\begin{array}{l}12.5 \% \\
-10.1 \%\end{array}$ & $0.3 \%$ \\
\hline Neutrino interaction & $\begin{array}{l}3.4 \% \\
-3.5 \%\end{array}$ & $\begin{array}{l}3.7 \% \\
-3.7 \%\end{array}$ & $\begin{array}{l}1.6 \% \\
-1.5 \%\end{array}$ \\
\hline Detector response & $1.1 \%$ & $1.7 \%$ & $2.04 \%$ \\
\hline Total & $\begin{array}{l}13.2 \% \\
-11.0 \%\end{array}$ & $\begin{array}{l}13.1 \% \\
-10.9 \%\end{array}$ & $\begin{array}{l}2.6 \% \\
-2.6 \%\end{array}$ \\
\hline
\end{tabular}

Table 2. Summary of the systematic errors on the CC cross section.

\subsection{Results}

The measured flux-averaged $\mathrm{CC}$ inclusive cross section on $\mathrm{Fe}, \mathrm{CH}$ and their ratio are:

$$
\begin{aligned}
\sigma_{C C}^{F e} & =\left(1.444 \pm 0.002(\text { stat } .)_{-0.159}^{0.191}(\text { syst. })\right) \times 10^{-38} \mathrm{~cm}^{2} / \text { nucleon } \\
\sigma_{C C}^{C H} & =\left(1.379 \pm 0.009(\text { stat } .)_{-0.150}^{0.181}(\text { syst. })\right) \times 10^{-38} \mathrm{~cm}^{2} / \text { nucleon } \\
\frac{\sigma_{C C}^{F e}}{\sigma_{C C}^{C H}} & =1.047 \pm 0.007(\text { stat. })_{-0.027}^{0.028}(\text { syst } .)
\end{aligned}
$$

at the mean neutrino energy of $1.51 \mathrm{GeV}$. These predictions agree well with the predicted values from NEUT and GENIE as shown on Figure 3. The main difference between the two models is the axial mass, which is $1.21 \mathrm{GeV} . \mathrm{c}^{-2}$ for NEUT and $0.99 \mathrm{GeV} . \mathrm{c}^{-2}$ for GENIE. The cross section ratio on Fe to
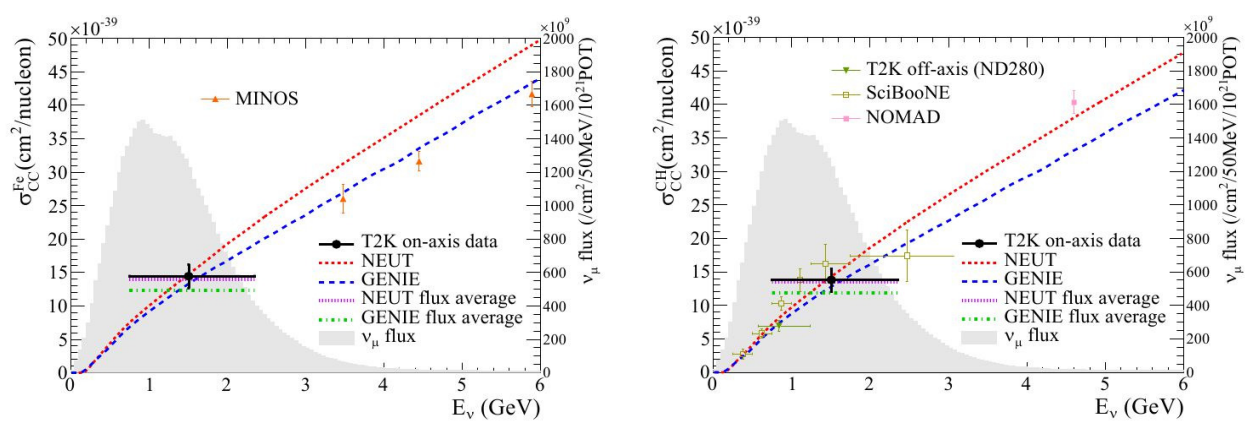

Figure 3. The inclusive $v_{\mu} \mathrm{CC}$ cross section on Fe (left) and $\mathrm{CH}$ (right) with predictions by NEUT and GENIE. The isoscalar corrections are not applied to our data predictions. Our data point is placed at the flux mean energy. The vertical error bar represents the total (statistical and systematic) uncertainty, and the horizontal bar represents $68 \%$ of the flux eacg side of the mean energy. The MINOS, SciBooNE, T2K ND280 and NOMAD results are also plotted [12],[13],[4],[14]. Because the isoscalar correction is applied to the MINOS data, it is expected to be shifted by about $-2 \%$.

$\mathrm{CH} 3 \%$ accuracy is also in agreement with the two models, which demonstrates the target dependence of nuclear effects is treated consistently in the models within a $3 \%$ accuracy. 


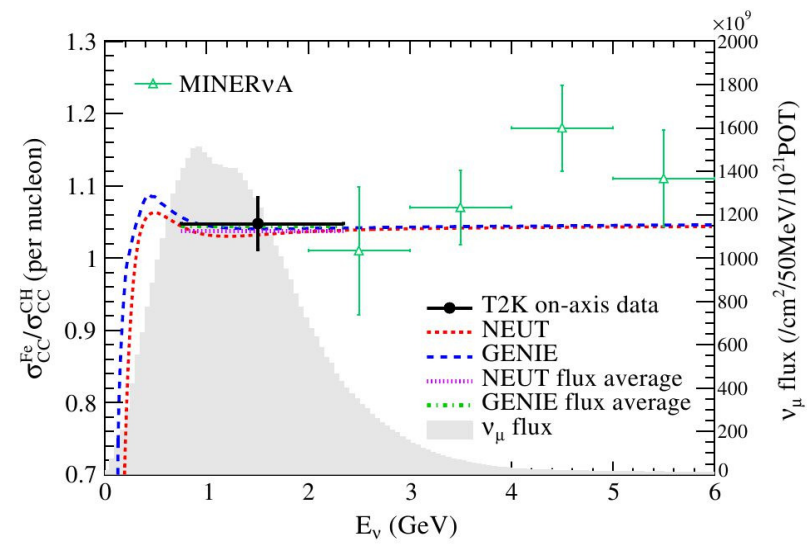

Figure 4. The inclusive $v_{\mu}$ charged current cross section ratio on Fe to $\mathrm{CH}$ with predictions by NEUT and GENIE. The isoscalar corrections are not applied to our data or predictions. Our data point is placed at the flux mean energy. The vertical error bar represents the total (statistical and systematic) uncertainty, and the horizontal bar represents $68 \%$ of the flux eacg side of the mean energy. The MINER $v$ A result is also plotted [15].

\section{Measurement of the $v_{\mu}$ cross section on hydrocarbon through quasi-elastic charged current interactions}

The quasi-elastic charged current (CCQE) interaction of neutrinos is the dominant interaction process at T2K's neutrino energy. The CCQE interaction is defined as:

$$
v_{\mu}+n \rightarrow \mu^{-}+p
$$

The measurement of this cross section on hydrocarbon is based on interactions in the PM, whose hydrocarbon is the dominant target (98.6\%). The CCQE cross section at two different neutrino energy points is measured based on the secondary muon properties. The INGRID horizontal module located downstream the PM is used for this purpose. A separation is done between secondary muons produced in PM but that propagate through the INGRID module ("through-going") and those which stop ("stopping") inside. For example, the event shown on Figure 2 corresponds to a through going event. These categories constitutes respectively the neutrino high $\left(<E_{v}>=1.94 \mathrm{GeV}, 1 \sigma \in[1.25,2.80]\right.$ $\mathrm{GeV})$ and low energy samples $\left(<E_{v}>=0.93 \mathrm{GeV}, 1 \sigma \in[0.54,1.31] \mathrm{GeV}\right)$. The cross section for each bin will be evaluated as in Eq 1.

\subsection{CCQE analysis}

\section{Event selection}

The primary event selection is based on the same PM selection that is described in Section 3 to identify the CC interactions. It provides a sample with 37.7\% CCQE purity. To increase the latter, a dedicated CCQE selection is applied on top of the CC selection. This CCQE selection is based on the final states of the CCQE: the number of tracks, the particle identification associated with each track and the kinematics of the event. A CCQE interaction is shown on Figure 2 and one expects an outgoing lepton in the final state and a possible proton if reconstructed. Therefore, it is required that a maximum of 
two tracks are reconstructed. The particle identification is then applied to each track, based on the energy deposition. For the one track sample, the track is required to be muon-like. For the two tracks sample, one should be muon-like and the other shouldn't be, in order to separate the protons from pions that are muon-like. Finally, for the two track sample, two kinematic cuts are applied to separate the CCQE from the remaining other $\mathrm{CC}$ interactions. The first cut is based on the opening angle between the two reconstructed tracks. Unlike CCQE, other CC produces at least three particles in the final state, of which only two are reconstructed. In the case of CCQE, two tracks are produced in the final state, of which are back-to-back in the centre of mass frame. For this reason, the opening angle between the two tracks tends to be higher for CCQE than for other CC interactions. Therefore, only two track sample events with an opening angle higher than $60^{\circ}$ are selected. On top of this, transverse momentum conservation requires the neutrino and outgoing tracks to be coplanar in the case of CCQE where two outgoing particles are produced (neglecting neutron momentum). This is not necessarily the case for the other $\mathrm{CC}$ interactions since only two of the outgoing tracks are reconstructed. The coplanarity is defined here as the angle between the cross product of the beam with the two tracks, namely:

$$
\gamma=\left(\overrightarrow{v_{\mu}} \times \overrightarrow{p_{1}}, \overrightarrow{v_{\mu}} \times \overrightarrow{p_{2}}\right)
$$

with $\vec{p}_{i}$ the unit vector of the i-th track. Three coplanar tracks have $\gamma=180^{\circ}$. The coplanarity between reconstructed tracks therefore then tends to be higher for CCQE interactions than for other CC. For this reason, only tracks with a coplanarity angle higher than $150^{\circ}$ are selected.

The neutrino flux, selection efficiency, background and number of target nucleons are evaluated similarly for CC interactions. The same data set is used. The details on the selection are summarised in Table 3 both for one, two-tracks and the combined sample for the two energy bins we introduced.

\begin{tabular}{|c|c|c|c|c|c|}
\hline \hline & $N_{\text {sel }}$ & $N_{\text {BG }}$ & $\phi$ & $N_{T}$ & $\epsilon$ \\
\hline One-track high energy & 5018.1 & 1073.7 & $2.025 \times 10^{13}$ & $8.452 \times 10^{28}$ & $18.8 \%$ \\
Two-track high energy & 1445.5 & 244.8 & $2.025 \times 10^{13}$ & $8.452 \times 10^{28}$ & $6.4 \%$ \\
Combined high energy & 6463.5 & 1318.5 & $2.025 \times 10^{13}$ & $8.452 \times 10^{28}$ & $25.1 \%$ \\
One-track low energy & 1456.8 & 479.2 & $1.885 \times 10^{13}$ & $8.452 \times 10^{28}$ & $5.3 \%$ \\
Two-track low energy & 408.9 & 155.2 & $1.885 \times 10^{13}$ & $8.452 \times 10^{28}$ & $2.0 \%$ \\
Combined low energy & 1865.8 & 634.5 & $1.885 \times 10^{13}$ & $8.452 \times 10^{28}$ & $7.3 \%$ \\
\hline \hline
\end{tabular}

Table 3. Summary of the candidate events that interacted through CCQE on hydrocarbon (PM). The selected background $N_{\mathrm{BG}}$, the intercepting neutrino flux in the detector fiducial volume $(\phi)$, the number of nucleon target (T) and the efficiency $\epsilon$ are also indicated for the two detectors.

\section{Systematics}

The systematic errors are separated into three sources: neutrino flux, neutrino interaction model and detector response. These errors are evaluated as for the inclusive CC measurement in Section 3, but independently for each sample (one track, two tracks, combined). A summary of each systematic error contribution is shown in Table 4, and exhibit an average 15\% error. It highlights that the dominant contribution comes from the neutrino flux errors as expected from any absolute cross section measurement. 


\begin{tabular}{|c|c|c|c|}
\hline \hline Source & One-track & Two-track & Combined \\
\hline Neutrino flux & $13.6 \%$ & $13.9 \%$ & $13.6 \%$ \\
Neutrino interaction & $-11.0 \%$ & $-11.3 \%$ & $-11.0 \%$ \\
Detector response & $7.4 \%$ & $8.7 \%$ & $5.9 \%$ \\
& $-5.6 \%$ & $-7.0 \%$ & $-4.1 \%$ \\
\hline Total & $1.0 \%$ & $4.6 \%$ & $3.6 \%$ \\
\hline \hline
\end{tabular}

Table 4. Summary of the systematic errors on CCQE cross section.

\subsection{Results}

The measurement of the CCQE cross sections on hydrocarbon from the different track samples of the high energy bin are:

$$
\begin{aligned}
& \sigma_{H E}^{1 \text { track }}=\left(1.229 \pm 0.022(\text { stat } .)_{-0.159}^{0.196}(\text { syst } .)\right) \times 10^{-38} \mathrm{~cm}^{2} / \text { nucleon } \\
& \sigma_{H E}^{2 \text { tracks }}=\left(1.098 \pm 0.035(\text { stat })_{-0.155}^{0.188}(\text { syst } .)\right) \times 10^{-38} \mathrm{~cm}^{2} / \text { nucleon } \\
& \sigma_{H E}^{\text {Comb. }}=\left(1.195 \pm 0.019(\text { stat } .)_{-0.147}^{0.182}(\text { syst. })\right) \times 10^{-38} \mathrm{~cm}^{2} / \text { nucleon }
\end{aligned}
$$

for neutrinos whose mean energy is $1.94 \mathrm{GeV}$ and where $68 \%$ of the events are located in the energy range $[1.25,2.80] \mathrm{GeV}$. As for the low energy bin, the results are:

$$
\begin{aligned}
\sigma_{L E}^{1 \text { track }} & =\left(1.163 \pm 0.045(\text { stat } .)_{-0.203}^{0.238}(\text { syst. })\right) \times 10^{-38} \mathrm{~cm}^{2} / \text { nucleon } \\
\sigma_{\text {LE }}^{2 \text { tracks }} & =\left(0.801 \pm 0.064(\text { stat. })_{-0.155}^{0.195}(\text { syst. })\right) \times 10^{-38} \mathrm{~cm}^{2} / \text { nucleon } \\
\sigma_{L E}^{\text {Comb. }} & =\left(1.064 \pm 0.037(\text { stat } .)_{-0.170}^{0.204}(\text { syst. })\right) \times 10^{-38} \mathrm{~cm}^{2} / \text { nucleon }
\end{aligned}
$$

for neutrinos whose mean energy is $0.93 \mathrm{GeV}$ and where $68 \%$ of the events are located in the energy range $[0.54,1.31] \mathrm{GeV}$. First, the comparisons with the GENIE and NEUT models are shown on Figure 5. One observes the agreement between the combined sample and the GENIE and NEUT model to be within $2 \sigma$. One observes the agreement with NEUT is significantly better, which tends to indicate a higher axial mass than $0.99 \mathrm{GeV} . \mathrm{c}^{-2}$ is preferred.

However, one observes the cross section results for the one track sample are always higher than the two track sample, and at more than $1 \sigma$ for the low energy sample. This unexpected disagreement can be understood as a difference between the nuclear effects for one and two tracks samples. One shows the main contribution to the systematics of the one and two track cross section ratio comes from the spectral function, which is not simulated in the default NEUT and taken as a systematic error. The result of the cross section implementing of the spectral function into NEUT is shown on Figure 6 and clearly shows a better agreement between the sample, which tends to show the nuclear effects aren't well simulated with a relativistic Fermi gas model. Finally, one shows most of the discrepancy between the one and two track sample vanishes when the meson exchanged currents between nucleons are added. This trend is shown on the right of Figure 6 and tends to confirm the differences between the one and two track sample illustrates an imperfect treatment of the nuclear effects with the relativistic Fermi gas model.

\section{Search for $v_{\mu}$ interaction through coherent pion scattering}

The neutrino interaction through coherent pion scattering has been observed at high energy (3 $300 \mathrm{GeV}$ ) in various experiments [18]. But, though there were several attempts in the SciBooNE [16] 


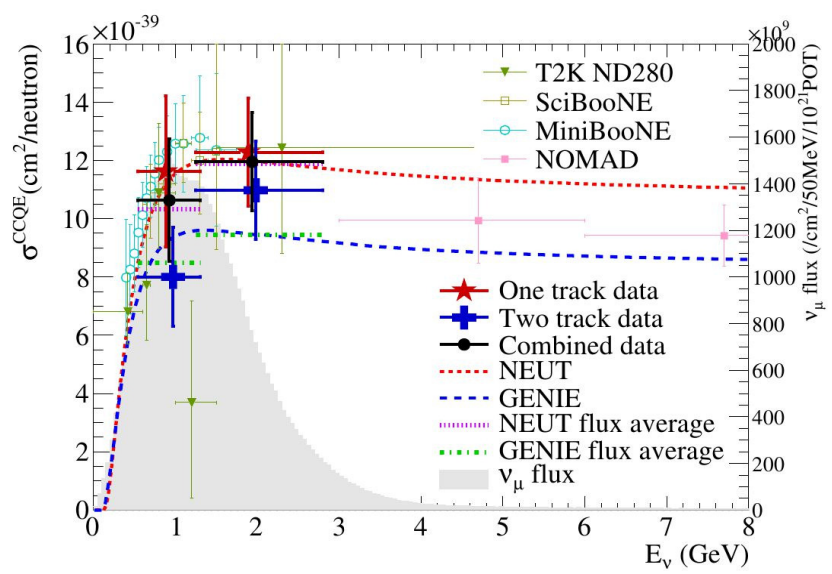

Figure 5. The CCQE cross section result with predictions by NEUT and GENIE. Our data point is placed at the flux mean energy. The vertical error bar represents the total (statistical and systematic) uncertainty, and the horizontal bar represents $68 \%$ of the flux at each side of the mean energy. SciBooNE [16], MiniBooNE [10], NOMAD [17] results are also plotted.
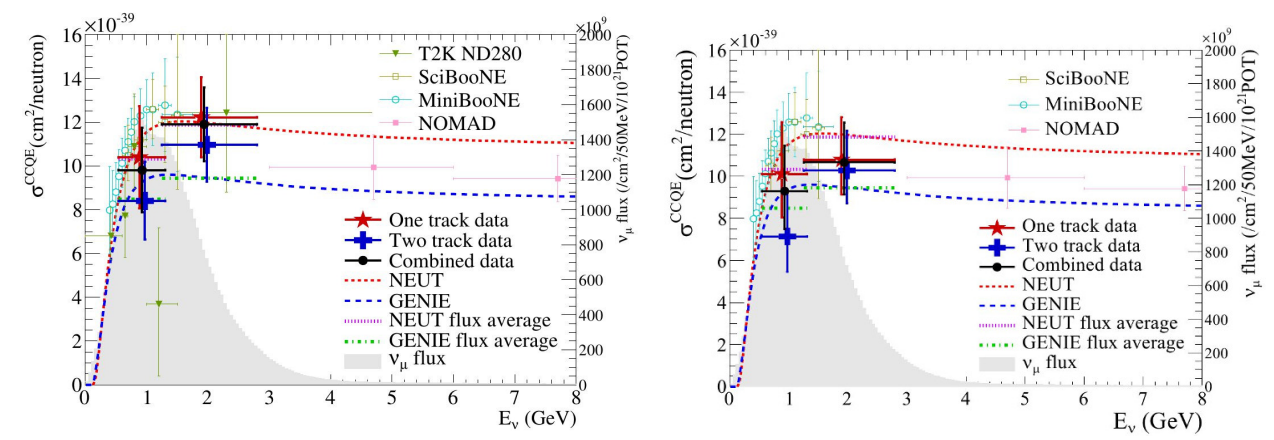

Figure 6. The CCQE cross section result with predictions by NEUT and GENIE. On the left is shown the result assuming a spectral function model. On the right, the meson exchanged currents are added to the model. Our data point is placed at the flux mean energy. The vertical error bar represents the total (statistical and systematic) uncertainty, and the horizontal bar represents $68 \%$ of the flux at each side of the mean energy. SciBooNE [16], MiniBooNE [10], NOMAD [17] results are also plotted.

and $\mathrm{K} 2 \mathrm{~K}$ [11] experiments, it was not observed at lower energy $E_{v} \sim 1 \mathrm{GeV}$. It is proposed to measure the cross section associated with this interaction on hydrocarbon, using the PM.

\subsection{CC coherent pion production analysis}

First, the CC selection (Section 3) is applied. On top of this, a dedicated CC coherent pion selection is used. The coherent pion production generally produces a muon and a pion in the final state. Therefore, a cut selecting only events with two reconstructed tracks is used. Then, the PID presented in Section 4 is used to identify the muon and pion tracks. To discriminate the coherent pion production from 
CCQE or resonant pion production, a kinematic cut is then applied. The coherent pion production generally implies very small momentum transfered from the neutrino to the nucleus (thus not seeing the nuclear structure). Therefore, one expects the muon to be scattered almost in the same direction as the incoming neutrino. It is required that the muon track has an angle less than $15^{\circ}$ with the neutrino beam direction. Finally, a cut on the vertex activity is used. The very low momentum transferred for coherent pion production implies small nuclear effects and vertex activity. For this reason, the energy deposit around the vertex is required to be lower than $25 \mathrm{MeV}$ which removes most of the interactions producing a pion through delta resonance.

\section{Systematics}

The systematic errors are separated into three sources: neutrino flux, neutrino interaction model and detector response. These errors are evaluated as for the inclusive $\mathrm{CC}$ measurement in Section 3. A summary of each systematic error contribution is shown in Table 4, and exhibit a large error $>65 \%$.

\begin{tabular}{|c|c|}
\hline \hline Source & Error \\
\hline Neutrino flux & $43.2 \%$ \\
$-35.7 \%$ \\
Neutrino interaction & $43.2 \%$ \\
Detector response & $30.0 \%$ \\
\hline Total & $68.4 \%$ \\
\hline
\end{tabular}

Table 5. Summary of the systematic errors on the cross section of CC coherent pion production.

\subsection{Results}

The measured $\mathrm{CC}$ coherent pion production cross section on hydrocarbon is:

$$
\sigma_{\text {CCcoh. } \pi}=\left(1.027 \pm 0.245(\text { stat } .)_{-0.677}^{0.703}(\text { syst. })\right) \times 10^{-39} \mathrm{~cm}^{2} / \text { nucleon }
$$

for neutrinos whose mean energy is $1.51 \mathrm{GeV}$ and whose $68 \%$ energy range is [0.75,2.35] GeV. The significance of the signal excess is $1.5 \sigma$ and therefore, we cannot make a conclusion about the existence of CC coherent pion production in this energy range. This result is shown on Figure 7, along with the comparison with NEUT and GENIE. Both generators use the Rein and Seghal model [19] but GENIE uses a more recent version tuned to K2K SciBar data. One observes the agreement between our data and GENIE is well within $1 \sigma$, which isn't the case for NEUT.

\section{Conclusions and perspectives}

The INGRID detector and the PM has an important role in the reduction of systematic errors for $v_{e}$ appearance and $v_{\mu}$ disappearance. It provides measurements complementary to the ND280, over a different energy range (higher energy). In this proceeding, we have presented the results of flux averaged cross sections from CC interactions on iron and hydrocarbon and have shown no discrepancy with predictions. The measurement of CCQE cross section on hydrocarbon has indicated that a higher axial mass is preferred, and that a tension exists between the one and two tracks sample. The latter can be reduced using a spectral function model instead of the relativistic Fermi gas, and taking into account the meson exchanged currents. Finally, we have shown that no significant signal of a coherent 


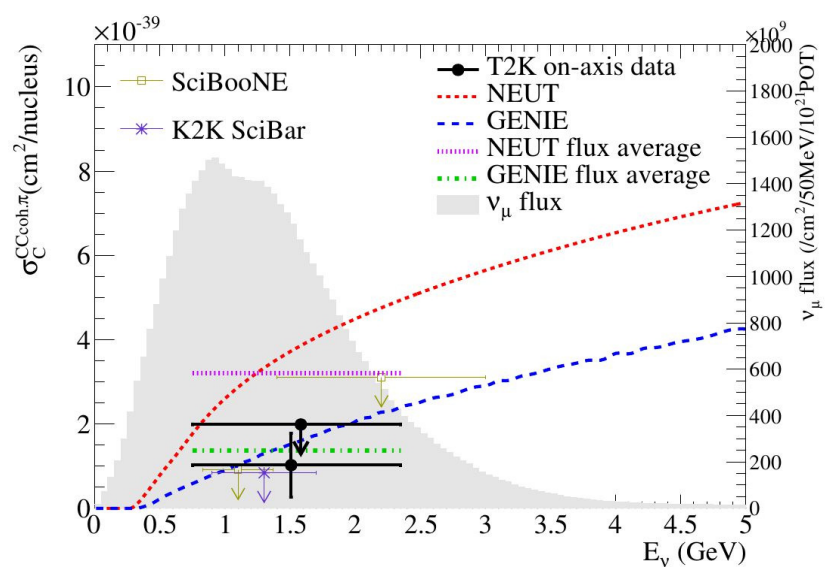

Figure 7. The CC coherent pion production cross section result with predictions by NEUT and GENIE. Our data point is placed at the flux mean energy. The vertical error bar represents the total (statistical and systematic) uncertainty, and the horizontal bar represents $68 \%$ of the flux at each side of the mean energy. SciBooNE [16] and K2K [11] results are also plotted. The upper black point along with the arrow represents the $90 \%$ confidence level upper limit on $\mathrm{CC}$ coherent pion production.

pion production through $\mathrm{CC}$ was observed.

In order to improve these measurements, differential cross sections are currently studied as the CC inclusive cross section with neutrino energy, or the double differential CCQE cross section with muon angle and momentum on hydrocarbon. In the latter, the model dependency is attempted to be minimal in order to minimise the associated systematics.

\section{References}

[1] K. Abe, J. Adam, H. Aihara, T. Akiri, C. Andreopoulos, S. Aoki, A. Ariga, T. Ariga, S. Assylbekov, D. Autiero et al., Physical review letters 112, 061802 (2014)

[2] F. An, J. Bai, A. Balantekin, H. Band, D. Beavis, W. Beriguete, M. Bishai, S. Blyth, K. Boddy, R. Brown et al., Physical Review Letters 108, 171803 (2012)

[3] J. Ahn, S. Chebotaryov, J. Choi, S. Choi, W. Choi, Y. Choi, H. Jang, J. Jang, E. Jeon, I. Jeong et al., Physical Review Letters 108, 191802 (2012)

[4] K. Abe, N. Abgrall, H. Aihara, T. Akiri, J. Albert, C. Andreopoulos, S. Aoki, A. Ariga, T. Ariga, S. Assylbekov et al., Physical Review D 87, 092003 (2013)

[5] N. Abgrall, A. Aduszkiewicz, B. Andrieu, T. Anticic, N. Antoniou, J. Argyriades, A. Asryan, B. Baatar, A. Blondel, J. Blumer et al., Physical Review C 84, 034604 (2011)

[6] N. Abgrall, A. Aduszkiewicz, T. Anticic, N. Antoniou, J. Argyriades, B. Baatar, A. Blondel, J. Blumer, M. Bogusz, L. Boldizsar et al., Physical Review C 85, 035210 (2012)

[7] T. Eichten, D. Haidt, J. Pattison, W. Venus, H. Wachsmuth, O. Wörz, T. Jones, B. Aubert, L. Chounet, P. Heusse et al., Nuclear Physics B 44, 333 (1972)

[8] J.V. Allaby, A.M. Thorndike, P. Duteil, J.P. Peigneux, J.P. Stroot, R. Meunier, A.M. Wetherell, E. Sacharidis, M. Spighel, F.G. Binon et al., Tech. rep., Cern (1970) 
[9] K. Abe, N. Abgrall, H. Aihara, T. Akiri, J. Albert, C. Andreopoulos, S. Aoki, A. Ariga, T. Ariga, S. Assylbekov et al., Physical Review D 87, 012001 (2013)

[10] A. Aguilar-Arevalo, C. Anderson, A. Bazarko, S. Brice, B. Brown, L. Bugel, J. Cao, L. Coney, J. Conrad, D. Cox et al., Physical Review D 81, 092005 (2010)

[11] K. Hiraide, J. Alcaraz-Aunion, S. Brice, L. Bugel, J. Catala-Perez, G. Cheng, J. Conrad, Z. Djurcic, U. Dore, D. Finley et al., Physical Review D 78, 112004 (2008)

[12] P. Adamson, C. Andreopoulos, K. Arms, R. Armstrong, D. Auty, D. Ayres, C. Backhouse, P. Barnes Jr, G. Barr, W. Barrett et al., Physical Review D 81, 072002 (2010)

[13] Y. Nakajima, J. Alcaraz-Aunion, S. Brice, L. Bugel, J. Catala-Perez, G. Cheng, J. Conrad, Z. Djurcic, U. Dore, D. Finley et al., Physical Review D 83, 012005 (2011)

[14] Q. Wu, S. Mishra, A. Godley, R. Petti, S. Alekhin, P. Astier, D. Autiero, A. Baldisseri, M. BaldoCeolin, M. Banner et al., Physics Letters B 660, 19 (2008)

[15] B. Tice, M. Datta, J. Mousseau, L. Aliaga, O. Altinok, M. Sazo, M. Betancourt, A. Bodek, A. Bravar, W. Brooks et al., arXiv preprint arXiv:1403.2103 (2014)

[16] Y. Kurimoto, J. Alcaraz-Aunion, S. Brice, L. Bugel, J. Catala-Perez, G. Cheng, J. Conrad, Z. Djurcic, U. Dore, D. Finley et al., Physical Review D 81, 033004 (2010)

[17] V. Lyubushkin, B. Popov, J. Kim, L. Camilleri, J.M. Levy, M. Mezzetto, D. Naumov, S. Alekhin, P. Astier, D. Autiero et al., The European Physical Journal C-Particles and Fields 63, 355 (2009)

[18] J. Beringer, J. Arguin, R. Barnett, K. Copic, O. Dahl, D. Groom, C. Lin, J. Lys, H. Murayama, C. Wohl et al., Physical Review D 86 (2012)

[19] D. Rein, L.M. Sehgal, Annals of Physics 133, 79 (1981) 